\title{
First Observation of the Doubly Charmed Baryon Decay $\Xi_{c c}^{++} \rightarrow \Xi_{c}^{+} \pi^{+}$
}

\author{
R. Aaij et al. \\ (LHCb Collaboration)
}

(Received 9 July 2018; revised manuscript received 1 August 2018; published 17 October 2018)

\begin{abstract}
The doubly charmed baryon decay $\Xi_{c c}^{++} \rightarrow \Xi_{c}^{+} \pi^{+}$is observed for the first time, with a statistical significance of 5.9\%, confirming a recent observation of the baryon in the $\Lambda_{c}^{+} K^{-} \pi^{+} \pi^{+}$final state. The data sample used corresponds to an integrated luminosity of $1.7 \mathrm{fb}^{-1}$, collected by the LHCb experiment in $p p$ collisions at a center-of-mass energy of $13 \mathrm{TeV}$. The $\Xi_{c c}^{++}$mass is measured to be $3620.6 \pm 1.5$ (stat) \pm 0.4 (syst) $\pm 0.3\left(\Xi_{c}^{+}\right) \mathrm{MeV} / c^{2}$ and is consistent with the previous result. The ratio of branching fractions between the decay modes is measured to be $\left[\mathcal{B}\left(\Xi_{c c}^{++} \rightarrow \Xi_{c}^{+} \pi^{+}\right) \times \mathcal{B}\left(\Xi_{c}^{+} \rightarrow p K^{-} \pi^{+}\right)\right] /\left[\mathcal{B}\left(\Xi_{c c}^{++} \rightarrow\right.\right.$ $\left.\left.\Lambda_{c}^{+} K^{-} \pi^{+} \pi^{+}\right) \times \mathcal{B}\left(\Lambda_{c}^{+} \rightarrow p K^{-} \pi^{+}\right)\right]=0.035 \pm 0.009$ (stat) \pm 0.003 (syst).
\end{abstract}

DOI: $10.1103 /$ PhysRevLett.121.162002

The recent observation by the LHCb Collaboration [1] of a new state that is consistent with the doubly charmed baryon $\Xi_{c c}^{++}$opens a new field of research studying the properties of baryons containing two heavy quarks, providing a unique environment for testing models of quantum chromodynamics. In studies of a sample of $\Xi_{c c}^{++}$decays to the final state $\Lambda_{c}^{+} K^{-} \pi^{+} \pi^{+}$, with $\Lambda_{c}^{+} \rightarrow p K^{-} \pi^{+}$, its mass was found to be $3621.40 \pm 0.72$ (stat) \pm 0.27 (syst) \pm $0.14\left(\Lambda_{c}^{+}\right) \mathrm{MeV} / c^{2}$ [1], and its lifetime was measured to be $0.256_{-0.022}^{+0.024}$ (stat) \pm 0.014 (syst) ps [2]. (The inclusion of charge-conjugate processes is implied throughout.) The measured lifetime firmly establishes its weakly decaying nature. Searching for new decay modes is the next critical step toward understanding the dynamics of weak decays of doubly heavy baryons, which may differ significantly from those of singly heavy hadrons due to interference between decay amplitudes of the two heavy quarks. The process $\Xi_{c c}^{++} \rightarrow \Xi_{c}^{+} \pi^{+}$has been predicted to have a sizable branching fraction $[3,4]$, making it a promising final state in which to seek confirmation of the previous observation.

This Letter reports the first observation of the decay $\Xi_{c c}^{++} \rightarrow \Xi_{c}^{+} \pi^{+}$, which proceeds predominantly via the treelevel amplitude represented by the Feynman diagram shown in Fig. 1. The $\Xi_{c}^{+}$baryon is reconstructed in its Cabibbo-suppressed decay to $p K^{-} \pi^{+}$. The data sample used consists of $p p$ collisions at a center-of-mass energy of $13 \mathrm{TeV}$ collected by the $\mathrm{LHCb}$ experiment in 2016, corresponding to an integrated luminosity of $1.7 \mathrm{fb}^{-1}$. A measurement of the $\Xi_{c c}^{++}$mass with this sample is

*Full author list given at the end of the article.

Published by the American Physical Society under the terms of the Creative Commons Attribution 4.0 International license. Further distribution of this work must maintain attribution to the author(s) and the published article's title, journal citation, and DOI. Funded by SCOAP ${ }^{3}$. presented, and the ratio of the total branching fractions $\mathcal{R}(\mathcal{B})$ between the decays $\Xi_{c c}^{++} \rightarrow \Xi_{c}^{+}\left(\rightarrow p K^{-} \pi^{+}\right) \pi^{+}$and $\Xi_{c c}^{++} \rightarrow \Lambda_{c}^{+}\left(\rightarrow p K^{-} \pi^{+}\right) K^{-} \pi^{+} \pi^{+}$is determined.

The $\mathrm{LHCb}$ detector $[5,6]$ is a single-arm forward spectrometer covering the pseudorapidity range $2<\eta<5$, designed for the study of particles containing $b$ or $c$ quarks. The detector includes a high-precision tracking system consisting of a silicon-strip vertex detector [7] surrounding the $p p$ interaction region that allows $c$ and $b$ hadrons to be identified from their typical long flight distance; a tracking system [8] that provides a measurement of momentum $p$ of charged particles; two ring-imaging Cherenkov detectors [9] that discriminate between different species of charged hadrons; a calorimeter system consisting of scintillatingpad and preshower detectors, an electromagnetic calorimeter and a hadronic calorimeter, to identify photons, electrons and hadrons; and a muon system composed of alternating layers of iron and multiwire proportional chambers [10] to identify muons. The online event selection is performed by a trigger [11], which consists of a hardware stage, based on information from the calorimeter and muon systems [12], followed by a software stage, which applies a full event reconstruction incorporating real-time alignment and calibration of the detector [13]. The same alignment and calibration information is propagated to the offline reconstruction, ensuring consistent and high-quality particle identification information between the trigger and offline software. The identical performance of the online and offline reconstruction offers

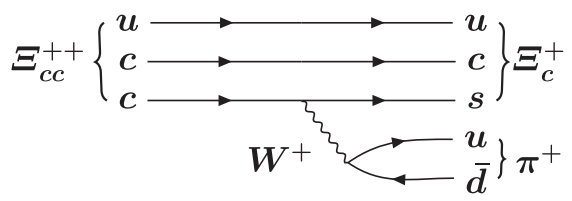

FIG. 1. Dominant Feynman diagram contributing to the decay $\Xi_{c c}^{++} \rightarrow \Xi_{c}^{+} \pi^{+}$. 
the opportunity to perform physics analyses directly using candidates reconstructed in the trigger which is done in the present analysis.

Simulation is required to model the effects of the detector acceptance and the imposed selection requirements. In the simulation, $p p$ collisions are generated using PYTHIA [14] with a specific LHCb configuration [15]. A dedicated generator, GENXICC2.0 [16], is used to simulate $\Xi_{c c}^{++}$ baryon production. Decays of hadrons are described by EvTGEN [17], in which final-state radiation is generated using Рнотоs [18]. The interaction of the generated particles with the detector, and its response, are modeled using the GEANT4 toolkit [19] as described in Ref. [20].

The selection of $\Xi_{c c}^{++} \rightarrow \Xi_{c}^{+}\left(\rightarrow p K^{-} \pi^{+}\right) \pi^{+}$decays is designed to be as similar as possible to those of $\Xi_{c c}^{++} \rightarrow \Lambda_{c}^{+}\left(\rightarrow p K^{-} \pi^{+}\right) K^{-} \pi^{+} \pi^{+}$, described in Ref. [1]. Three charged particles identified as $p, K^{-}$, and $\pi^{+}$that form a good-quality vertex are combined to reconstruct a $\Xi_{c}^{+} \rightarrow p K^{-} \pi^{+}$candidate. The three particles are required to have transverse momenta in excess of $500 \mathrm{MeV} / c$ and be inconsistent with originating from any primary vertex $(\mathrm{PV})$. The $\Xi_{c}^{+}$vertex is required to be displaced from any PV by a distance corresponding to a $\Xi_{c}^{+}$decay time greater than $0.15 \mathrm{ps}$, which corresponds to approximately twice the decay time resolution. The invariant mass of each $\Xi_{c}^{+}$candidate is required to be in the range 2450$2488 \mathrm{MeV} / c^{2}$, corresponding to approximately 6 times the $\Xi_{c}^{+}$mass resolution. An additional positively charged particle, which must be identified as a pion and have $p_{T}$ greater than $200 \mathrm{MeV} / c$, is then combined with the $\Xi_{c}^{+}$ candidate to form a $\Xi_{c c}^{++}$candidate. The $\Xi_{c}^{+} \pi^{+}$pair is required to form a vertex that is of good quality and is upstream of the $\Xi_{c}^{+}$vertex. The $\Xi_{c c}^{++}$candidate must have $p_{T}>2000 \mathrm{MeV} / c$ and be consistent with originating from a PV. The candidate is associated with the PV with respect to which it has the smallest impact parameter $\chi^{2}\left(\chi_{\mathrm{IP}}^{2}\right)$. The $\chi_{\mathrm{IP}}^{2}$ is defined as the difference in $\chi^{2}$ of the PV fit with and without the particle in question. To avoid contributions due to duplicate tracks, candidates are rejected if the angle between any pair of their final-state particles with the same charge is smaller than $0.5 \mathrm{mrad}$. Specific hardware trigger requirements are also applied, to increase the signal yield and simplify the study of the trigger efficiency. Candidates are retained only if the event contains large transverse energy deposits in the calorimeter arising from the decay products of the $\Xi_{c c}^{++}$candidate, or if the event contains activity either in the calorimeter or in the muon system from particles other than these decay products. Simulation shows that the efficiency for these additional requirements is above $90 \%$ for both two-body or four-body $\Xi_{c c}^{++}$ decay modes.

A multivariate selector based on the multilayer perceptron algorithm [21] is used to further suppress combinatorial backgrounds. To train the selector, simulated $\Xi_{c c}^{++} \rightarrow$ $\Xi_{c}^{+}\left(\rightarrow p K^{-} \pi^{+}\right) \pi^{+}$decays are used as a signal sample, and
0.3 million candidates from the upper sideband with invariant masses in the range $3800-4000 \mathrm{MeV} / c^{2}$ are used as a background sample. To reduce the effect of the $\Xi_{c}^{+}$mass resolution on the invariant-mass of the $\Xi_{c c}^{++}$candidates, an alternative evaluation of the invariant mass is used, $m\left(\Xi_{c}^{+} \pi^{+}\right) \equiv M\left(\Xi_{c}^{+} \pi^{+}\right)-M\left(\left[p K^{-} \pi^{+}\right]_{\Xi_{c}^{+}}\right)+M_{\mathrm{PDG}}\left(\Xi_{c}^{+}\right)$, where $M\left(\Xi_{c}^{+} \pi^{+}\right)$and $M\left(\left[p K^{-} \pi^{+}\right]_{\Xi_{c}^{+}}\right)$are the reconstructed masses of the $\Xi_{c c}^{++}$and $\Xi_{c}^{+}$candidates, and $M_{\mathrm{PDG}}\left(\Xi_{c}^{+}\right)$is the known value of the $\Xi_{c}^{+}$mass [22].

The input variables used in the multivariate selector are chosen based on their discrimination power between signal and background candidates. Three different types of variables are considered in the training. The first type of variables are the kinematic information of particles, including the $p_{T}$ of each of the four final-state particles and of the $\Xi_{c}^{+}$and $\Xi_{c c}^{++}$candidates, and the angle between the $\Xi_{c c}^{++}$ momentum vector and the displacement vector from the PV to the $\Xi_{c c}^{++}$decay vertex. The second type of variables are the vertex fitting qualities, including the $\chi^{2}$ per degree of freedom of the $\Xi_{c}^{+}$and $\Xi_{c c}^{++}$vertex fits, and the $\chi^{2}$ per degree of freedom of a kinematic refit [23] of the $\Xi_{c c}^{++} \rightarrow$ $\Xi_{c}^{+}\left(\rightarrow p K^{-} \pi^{+}\right) \pi^{+}$decay chain that requires the $\Xi_{c c}^{++}$to originate from its PV. The third type of variables are related to the lifetime, including the $\chi_{\text {IP }}^{2}$ of each of the four finalstate particles and of the $\Xi_{c}^{+}$and $\Xi_{c c}^{++}$candidates with respect to their associated $\mathrm{PV}$, the sum of the $\chi_{\mathrm{IP}}^{2}$ of the four final-state particles, and the flight distance $\chi^{2}$ of the $\Xi_{c}^{+}$and $\Xi_{c c}^{++}$candidates. The flight distance $\chi^{2}$ is defined as the $\chi^{2}$ of the hypothesis that the decay vertex of the candidate coincides with its associated PV.

Candidates are retained only if the multivariate-selector output exceeds a certain threshold. This threshold is chosen to maximize the expected value of the figure of merit $\varepsilon /\left(\frac{5}{2}+\sqrt{N_{\mathrm{B}}}\right)$ [24]. Here, $\varepsilon$ is the estimated signal efficiency and $N_{\mathrm{B}}$ is the expected number of background candidates under the signal peak in the $\Xi_{c c}^{++}$mass distribution, after the selection. The quantity $N_{\mathrm{B}}$ is determined, assuming an exponential shape for the background, from the number of $\Xi_{c}^{+} \pi^{+}$candidates in the mass region of $3800-4000 \mathrm{MeV} / c^{2}$, scaled to a signal region centered at a mass of $3620 \mathrm{MeV} / c^{2}$ and with a width of $30 \mathrm{MeV} / c^{2}$. This corresponds to approximately 5 times the expected $\Xi_{c c}^{++}$mass resolution. To test for potential biases in the multivariate selection or other misreconstruction effects, the same selection criteria are applied to control samples of data consisting of $\Xi_{c}^{+} \pi^{+}$candidates in the $\Xi_{c}^{+}$sideband regions and of wrong-sign combination $\Xi_{c}^{+} \pi^{-}$. No peaking structure is visible in either sample.

Figure 2 (left) shows the distribution of invariant masses of $\Xi_{c c}^{++}$candidates, $m\left(\Xi_{c}^{+} \pi^{+}\right)$, after applying the complete selection. The contribution from events containing multiple signal candidates is found to be less than $1 \%$; all of these candidates are included in the fit. A signal is visible at a mass of approximately $3620 \mathrm{MeV} / c^{2}$, in the vicinity of the 

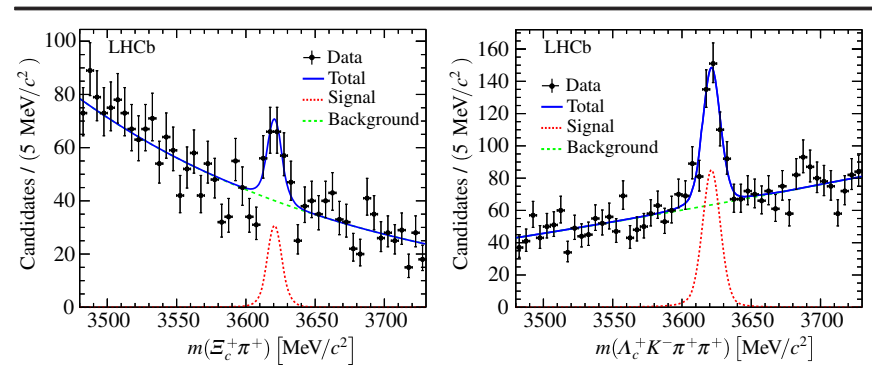

FIG. 2. Invariant-mass distribution of the $\Xi_{c c}^{++} \rightarrow \Xi_{c}^{+} \pi^{+}$(left) and $\Xi_{c c}^{++} \rightarrow \Lambda_{c}^{+} K^{-} \pi^{+} \pi^{+}$(right) candidates with result of the fit overlaid. The black points represent the data, the dotted (red) line represents the signal contribution, and the dashed (green) line represents the combinational background.

previous LHCb $\Xi_{c c}^{++}$baryon observation [1]. The mass distribution is fitted with an unbinned extended maximumlikelihood method to measure the properties of this structure. The peak is described by an empirical model, consisting of a Gaussian function and a modified Gaussian function with power-law tails on both sides [25] and with the same mean value. All tail parameters are fixed to values obtained from a fit to simulated signal events, while the parameters corresponding to the mass and the mass resolution are varied in the fit. The background shape is described by an exponential function. The resulting signal yield is $91 \pm 20$ and the mass value is $3620.7 \pm 1.5 \mathrm{MeV} / c^{2}$, where the uncertainties are statistical only. The mass is fully consistent with the value measured in the $\Xi_{c c}^{++} \rightarrow \Lambda_{c}^{+} K^{-} \pi^{+} \pi^{+}$decay channel [1], and the resolution determined by the fit is consistent with expectations based on known detector performance. The local statistical significance of the signal, evaluated by taking the likelihood ratio corresponding to fits that include and exclude the signal component, is found to be $5.9 \sigma$, thus confirming the observation reported in Ref. [1].

The invariant-mass distribution for the reference mode $\Xi_{c c}^{++} \rightarrow \Lambda_{c}^{+} K^{-} \pi^{+} \pi^{+}$is shown in Fig. 2 (right). The selection used to obtain this sample is identical to that of the previous analysis [1], except for the additional requirements on the hardware trigger. An extended unbinned maximum-likelihood fit to the invariant-mass distribution returns a signal yield of $289 \pm 35$ for the reference mode.

The branching fraction ratio, $\mathcal{R}(\mathcal{B})$, between the decays $\Xi_{c c}^{++} \rightarrow \Xi_{c}^{+}\left(\rightarrow p K^{-} \pi^{+}\right) \pi^{+} \quad$ and $\quad \Xi_{c c}^{++} \rightarrow \Lambda_{c}^{+}\left(\rightarrow p K^{-} \pi^{+}\right)$ $K^{-} \pi^{+} \pi^{+}$is defined as

$$
\begin{aligned}
\mathcal{R}(\mathcal{B}) & \equiv \frac{\mathcal{B}\left(\Xi_{c c}^{++} \rightarrow \Xi_{c}^{+} \pi^{+}\right) \times \mathcal{B}\left(\Xi_{c}^{+} \rightarrow p K^{-} \pi^{+}\right)}{\mathcal{B}\left(\Xi_{c c}^{++} \rightarrow \Lambda_{c}^{+} K^{-} \pi^{+} \pi^{+}\right) \times \mathcal{B}\left(\Lambda_{c}^{+} \rightarrow p K^{-} \pi^{+}\right)} \\
& =\frac{r_{N}}{r_{\varepsilon}},
\end{aligned}
$$

where $r_{N}$ is the ratio of $\Xi_{c c}^{++}$yields between the signal and reference decay modes, and $r_{\varepsilon}$ is the ratio of total efficiencies between the two modes. In each case, the total efficiency includes the effects of the geometrical acceptance, trigger, reconstruction, and selection. Each contribution to the efficiency ratio is evaluated with simulation, calibrated with data when possible, as described in the following. The combined efficiency of the reconstruction and the selection, excluding the hardwaretrigger requirement, is determined from fully simulated signal samples in which the tracking [26] and particleidentification efficiencies are corrected using control samples. The correction to the efficiency ratio of the $\Xi_{c c}^{++} \rightarrow$ $\Lambda_{c}^{+} K^{-} \pi^{+} \pi^{+}$and $\Xi_{c c}^{++} \rightarrow \Xi_{c}^{+} \pi^{+}$channels is determined to be $0.983 \pm 0.007$ for the tracking efficiency, and $1.050 \pm$ 0.020 for the particle-identification efficiency. The hardware-trigger efficiency ratio is estimated from fully simu-

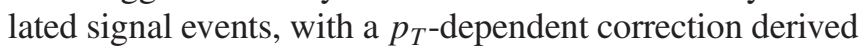
from data using $\Lambda_{b}^{0} \rightarrow \Lambda_{c}^{+}\left(\rightarrow p K^{-} \pi^{+}\right) \pi^{-} \pi^{+} \pi^{-}$and $\Lambda_{b}^{0} \rightarrow$ $\Lambda_{c}^{+}\left(\rightarrow p K^{-} \pi^{+}\right) \pi^{-}$decays, which are required to pass the same trigger selection as the $\Xi_{c c}^{++}$candidates. These two decay channels have similar final states and decay topologies as the signal. The total relative efficiency is determined to be $r_{\varepsilon}=0.110 \pm 0.002$, where the uncertainty comes from the limited size of the simulation sample and is accounted as a systematic uncertainty. To validate this procedure, the ratio of branching fractions of the decays $\Lambda_{b}^{0} \rightarrow \Lambda_{c}^{+}\left(\rightarrow p K^{-} \pi^{+}\right) \pi^{-}$and $\Lambda_{b}^{0} \rightarrow \Lambda_{c}^{+}\left(\rightarrow p K^{-} \pi^{+}\right) \pi^{-} \pi^{+} \pi^{-}$ is measured using the same data sample, resulting in a value $0.83 \pm 0.05$ (statistical uncertainty only) which agrees with the previous $\mathrm{LHCb}$ result of $0.70 \pm 0.10$ [27].

The main sources of systematic uncertainty that affect the measurements of the $\Xi_{c c}^{++}$mass are summarized in Table I. Samples of $J / \psi \rightarrow \mu^{+} \mu^{-}$and $B^{+} \rightarrow J / \psi K^{+}$ decays $[28,29]$ are used to calibrate the reconstructed momentum of charged particles, which affects the reconstructed mass of signal. The maximum difference between the correction factors determined with the above-mentioned decays is found to be $0.03 \%$, which corresponds to a systematic uncertainty of $0.38 \mathrm{MeV} / c^{2}$ on the measured $\Xi_{c c}^{++}$mass. The signal selection efficiency increases with the $\Xi_{c c}^{++}$decay time; combined with a correlation between the reconstructed mass and the reconstructed decay time, this induces a positive bias on the masses of both $\Xi_{c c}^{++}$and $\Xi_{c}^{+}$candidates. The effect is studied with simulation and

TABLE I. Systematic uncertainties on the measurement of the $\Xi_{c c}^{++}$mass and of the ratio of branching fractions $\mathcal{R}(\mathcal{B})$ between the $\Xi_{c c}^{++} \rightarrow \Xi_{c}^{+} \pi^{+}$and the $\Xi_{c c}^{++} \rightarrow \Lambda_{c}^{+} K^{-} \pi^{+} \pi^{+}$decay modes.

\begin{tabular}{lcc}
\hline \hline Source & Mass $\left[\mathrm{MeV} / c^{2}\right]$ & $\mathcal{R}(\mathcal{B})[\%]$ \\
\hline Momentum calibration & 0.38 & $\ldots$ \\
Selection bias correction & 0.10 & $\ldots$ \\
Fit model & 0.05 & 5.2 \\
Relative efficiency & $\ldots$ & 6.5 \\
Simulation modelling & $\ldots$ & 1.2 \\
Selection & $\ldots$ & 0.7 \\
Sum in quadrature & 0.40 & 8.5 \\
\hline \hline
\end{tabular}


the bias on the measured $\Xi_{c c}^{++}$mass is found to be $+0.10 \pm 0.10 \mathrm{MeV} / c^{2}$, where the uncertainty is due to the limited size of the simulated samples. A correction to the $\Xi_{c c}^{++}$mass of $-0.10 \mathrm{MeV} / c^{2}$ is therefore applied, and a systematic uncertainty of $0.10 \mathrm{MeV} / c^{2}$ assigned. The dependence of this bias on the $\Xi_{c c}^{++}$lifetime is studied by weighting simulated events to different lifetime hypotheses; the change is found to be negligible for the measured $\Xi_{c c}^{++}$lifetime [2]. The description of the final-state radiation in simulation [18] can also cause a bias in the measured mass, which is estimated with pseudoexperiments. The correction is determined to be $+0.03 \mathrm{MeV} / c^{2}$, with a negligible uncertainty. The impact of the model used to fit the invariant-mass distribution on the measured mass is estimated by varying the shape parameters that are fixed according to simulation, using alternative signal and background models, and performing the fits over different mass ranges. The largest variation in the fitted $\Xi_{c c}^{++}$masses, $0.05 \mathrm{MeV} / c^{2}$, is taken as a systematic uncertainty. The current known value for the $\Xi_{c}^{+}$mass [22] is used to compute the invariant-mass $m\left(\Xi_{c}^{+} \pi^{+}\right)$of the $\Xi_{c c}^{++}$candidate. Its uncertainty $0.30 \mathrm{MeV} / c^{2}$ is assigned as a systematic uncertainty on the $\Xi_{c c}^{++}$mass.

The systematic uncertainties on the ratio $\mathcal{R}(\mathcal{B})$ are listed in Table I and are described as follows. The alternative fit models mentioned above result in different values of the ratio $r_{N}$. The largest relative deviation measured $5.2 \%$ is assigned as a systematic uncertainty on $\mathcal{R}(\mathcal{B})$. The relative efficiency of the tracking, particle identification, and trigger are estimated using control samples, whose statistical uncertainties are taken as a systematic uncertainty on $\mathcal{R}(\mathcal{B})$. An additional uncertainty of $4.1 \%$ is assigned on the track-reconstruction efficiency due to uncertainties on the material budget of the detector and the modeling of hadronic interaction with the detector material. The particle-identification efficiency is determined in bins of particle momentum and pseudorapidity using control samples. The size of the bins is increased or decreased by a factor of 2 and the largest deviation on $\mathcal{R}(\mathcal{B})$ is assigned as systematic uncertainty related to the binning. An additional uncertainty of $4.2 \%$ on the hardware trigger efficiency is determined from the $\Lambda_{b}^{0}$ control samples described above, including a statistical uncertainty from the limited sample size, and an uncertainty that is determined by testing the procedure in simulation and taking the deviation as a systematic uncertainty. Combining the systematic uncertainties on the efficiency mentioned above, a systematic uncertainty of $6.5 \%$ on $\mathcal{R}(\mathcal{B})$ is assigned. Uncertainties from the $\Xi_{c c}^{++}$mass, lifetime, and production spectra are investigated, and $1.2 \%$ is assigned as a systematic uncertainty. Different requirements on the $\Xi_{c c}^{++} p_{T}$ are applied to select the $\Xi_{c c}^{++} \rightarrow \Xi_{c}^{+} \pi^{+}$and $\Xi_{c c}^{++} \rightarrow \Lambda_{c}^{+} K^{-} \pi^{+} \pi^{+}$decays, and this may cause a bias if the $p_{T}$ distribution of simulated $\Xi_{c c}^{++}$differs from that in data. To assess the size of this effect, the measurement is repeated applying the same $p_{T}$ requirement to both modes. The difference in $\mathcal{R}(\mathcal{B})$ is found to be $0.7 \%$. A separate measurement carried out with a cut-based selection gives a consistent result.

The value of $\mathcal{R}(\mathcal{B})$ is measured to be $0.035 \pm$ 0.009 (stat) \pm 0.003 (syst) and the $\Xi_{c c}^{++}$mass is measured to be $3620.6 \pm 1.5$ (stat) \pm 0.4 (syst) $\pm 0.3\left(\Xi_{c}^{+}\right) \mathrm{MeV} / c^{2}$, which is consistent with the mass measured in the final state $\Lambda_{c}^{+} K^{-} \pi^{+} \pi^{+}, \quad 3621.40 \pm 0.72$ (stat) \pm 0.27 (syst) \pm $0.14\left(\Lambda_{c}^{+}\right) \mathrm{MeV} / c^{2}$ [1]. Averaging over the two measurements, the $\Xi_{c c}^{++}$mass is determined to be $3621.24 \pm$ 0.65 (stat) \pm 0.31 (syst) $\mathrm{MeV} / c^{2}$ (see the Supplemental Material [30] for the comparisons between the measured $\Xi_{c c}^{++}$masses and combined result). The combination is performed using the best linear unbiased estimate method $[31,32]$. In the combination, the systematic uncertainties are assumed to be uncorrelated except for the momentum scale calibration.

In summary, a new decay mode of the doubly charmed baryon $\Xi_{c c}^{++} \rightarrow \Xi_{c}^{+} \pi^{+}$is observed with a statistical significance of $5.9 \sigma$ in a data sample of $p p$ collisions collected by the LHCb experiment at a center-of-mass energy of $\sqrt{s}=13 \mathrm{TeV}$. The $\Xi_{c c}^{++}$mass is consistent with the previous $\mathrm{LHCb}$ result [1] and with most theoretical calculations of the $\Xi_{c c}^{++}$mass (see, e.g., Ref. [33]). The ratio of the total branching fractions between this decay $\left(\Xi_{c c}^{++} \rightarrow \Xi_{c}^{+} \pi^{+}\right)$and the reference mode $\left(\Xi_{c c}^{++} \rightarrow \Lambda_{c}^{+} K^{-} \pi^{+} \pi^{+}\right)$is consistent with the prediction of Ref. [4], which, however, has large uncertainties. Therefore, this measurement provides important information toward an improved understanding of the decays of doubly charmed baryons.

We thank Chao-Hsi Chang, Cai-Dian Lü, Wei Wang, Xing-Gang $\mathrm{Wu}$, and $\mathrm{Fu}-\mathrm{Sheng} \mathrm{Yu}$ for frequent and interesting discussions on the production and decays of doubleheavy-flavor baryons. We express our gratitude to our colleagues in the CERN accelerator departments for the excellent performance of the LHC. We thank the technical and administrative staff at the LHCb institutes. We acknowledge support from CERN and from the national agencies: CAPES, CNPq, FAPERJ and FINEP (Brazil); MOST and NSFC (China); CNRS/IN2P3 (France); BMBF, DFG and MPG (Germany); INFN (Italy); NWO (Netherlands); MNiSW and NCN (Poland); MEN/IFA (Romania); MinES and FASO (Russia); MinECo (Spain); SNSF and SER (Switzerland); NASU (Ukraine); STFC (United Kingdom); NSF (USA). We acknowledge the computing resources that are provided by CERN, IN2P3 (France), KIT and DESY (Germany), INFN (Italy), SURF (Netherlands), PIC (Spain), GridPP (United Kingdom), RRCKI and Yandex LLC (Russia), CSCS (Switzerland), IFIN-HH (Romania), CBPF (Brazil), PL-GRID (Poland) and OSC (USA). We are indebted to the communities behind the multiple open-source software packages on which we depend. Individual groups or members have received support from AvH Foundation 
(Germany); EPLANET, Marie Skłodowska-Curie Actions and ERC (European Union); ANR, Labex P2IO and OCEVU, and Région Auvergne-Rhóne-Alpes (France); Key Research Program of Frontier Sciences of CAS, CAS PIFI, and the Thousand Talents Program (China); RFBR, RSF and Yandex LLC (Russia); GVA, XuntaGal and GENCAT (Spain); Herchel Smith Fund, the Royal Society, the English-Speaking Union and the Leverhulme Trust (United Kingdom); Laboratory Directed Research and Development program of LANL (USA).

[1] R. Aaij et al. (LHCb Collaboration), Observation of the Doubly Charmed Baryon $\Xi_{c c}^{++}$, Phys. Rev. Lett. 119, 112001 (2017).

[2] R. Aaij et al. (LHCb Collaboration), Measurement of the Lifetime of the Doubly Charmed Baryon $\Xi_{c c}^{++}$, Phys. Rev. Lett. 121, 052002 (2018).

[3] N. Sharma and R. Dhir, Estimates of W-exchange contributions to $\Xi_{c c}$ decays, Phys. Rev. D 96, 113006 (2017).

[4] F.-S. Yu, H.-Y. Jiang, R.-H. Li, C.-D. Lü, W. Wang, and Z.-X. Zhao, Discovery potentials of doubly charmed baryons, Chin. Phys. C 42, 051001 (2018).

[5] A. A. Alves, Jr. et al. (LHCb Collaboration), The LHCb detector at the LHC, J. Instrum. 3, S08005 (2008).

[6] R. Aaij et al. (LHCb Collaboration), $\mathrm{LHCb}$ detector performance, Int. J. Mod. Phys. A 30, 1530022 (2015).

[7] R. Aaij et al., Performance of the LHCb Vertex Locator, J. Instrum. 9, P09007 (2014).

[8] R. Arink et al., Performance of the LHCb outer tracker, J. Instrum. 9, P01002 (2014).

[9] M. Adinolfi et al., Performance of the LHCb RICH detector at the LHC, Eur. Phys. J. C 73, 2431 (2013).

[10] A. A. Alves, Jr. et al., Performance of the LHCb muon system, J. Instrum. 8, P02022 (2013).

[11] R. Aaij et al., The LHCb trigger and its performance in 2011, J. Instrum. 8, P04022 (2013).

[12] F. Archilli et al., Performance of the muon identification at LHCb, J. Instrum. 8, P10020 (2013).

[13] G. Dujany and B. Storaci, Real-time alignment and calibration of the LHCb Detector in Run II, J. Phys. Conf. Ser. 664, 082010 (2015).

[14] T. Sjöstrand, S. Mrenna, and P. Skands, A brief introduction to PYTHIA 8.1, Comput. Phys. Commun. 178, 852 (2008); PYTHIA 6.4 physics and manual, J. High Energy Phys. 05 (2006) 026.

[15] I. Belyaev et al., Handling of the generation of primary events in Gauss, the LHCb simulation framework, J. Phys. Conf. Ser. 331, 032047 (2011).

[16] C.-H. Chang, J.-X. Wang, and X.-G. Wu, GENXICC: A generator for hadronic production of the double heavy baryons $\Xi_{c c}, \Xi_{b c}$ and $\Xi_{b b}$, Comput. Phys. Commun. 177, 467 (2007); GENXICC2.0: An upgraded version of the generator for hadronic production of double heavy baryons $\Xi_{c c}, \Xi_{b c}$ and $\Xi_{b b}$, Comput. Phys. Commun. 181, 1144 (2010).

[17] D. J. Lange, The EvtGen particle decay simulation package, Nucl. Instrum. Methods Phys. Res., Sect. A 462, 152 (2001).

[18] P. Golonka and Z. Was, PHOTOS Monte Carlo: A precision tool for QED corrections in $Z$ and $W$ decays, Eur. Phys. J. C 45, 97 (2006).

[19] J. Allison et al. (Geant4 Collaboration), Geant4 developments and applications, IEEE Trans. Nucl. Sci. 53, 270 (2006); S. Agostinelli et al. (Geant4 Collaboration), Geant4: A simulation toolkit, Nucl. Instrum. Methods Phys. Res., Sect. A 506, 250 (2003).

[20] M. Clemencic, G. Corti, S. Easo, C. R. Jones, S. Miglioranzi, M. Pappagallo, and P. Robbe, The LHCb simulation application, Gauss: Design, evolution and experience, J. Phys. Conf. Ser. 331, 032023 (2011).

[21] H. Voss, A. Hoecker, J. Stelzer, and F. Tegenfeldt, TMVA: Toolkit for Multivariate Data Analysis, Proc. Sci., ACAT2007 (2007) 040; A. Hoecker et al., TMVA 4: Toolkit for Multivariate Data Analysis, Users Guide, arXiv:physics/ 0703039 .

[22] M. Tanabashi et al. (Particle Data Group), Review of particle physics, Phys. Rev. D 98, 030001 (2018).

[23] W. D. Hulsbergen, Decay chain fitting with a Kalman filter, Nucl. Instrum. Methods Phys. Res., Sect. A 552, 566 (2005).

[24] G. Punzi, Sensitivity of searches for new signals and its optimization, eConf C030908, MODT002 (2003).

[25] T. Skwarnicki, A study of the radiative cascade transitions between the Upsilon-prime and Upsilon resonances, Ph.D. thesis, Institute of Nuclear Physics, Krakow, 1986, Report No. DESY-F31-86-02, http://inspirehep.net/record/230779/.

[26] R. Aaij et al. (LHCb Collaboration), Measurement of the track reconstruction efficiency at $\mathrm{LHCb}, \mathrm{J}$. Instrum. 10, P02007 (2015).

[27] R. Aaij et al. (LHCb Collaboration), Measurements of the branching fractions for $B_{(s)} \rightarrow D_{(s)} \pi \pi \pi$ and $\Lambda_{b}^{0} \rightarrow \Lambda_{c}^{+} \pi \pi \pi$, Phys. Rev. D 84, 092001 (2011); Erratum, Phys. Rev. D 85, 039904 (2012).

[28] R. Aaij et al. (LHCb Collaboration), Measurement of $b$-hadron masses, Phys. Lett. B 708, 241 (2012).

[29] R. Aaij et al. (LHCb Collaboration), Precision measurement of $D$ meson mass differences, J. High Energy Phys. 06 (2013) 065.

[30] See Supplemental Material at http://link.aps.org/ supplemental/10.1103/PhysRevLett.121.162002 for the combined $\Xi_{c c}^{++}$mass from two experimental measurements.

[31] L. Lyons, D. Gibaut, and P. Clifford, How to combine correlated estimates of a single physical quantity, Nucl. Instrum. Methods Phys. Res., Sect. A 270, 110 (1988).

[32] A. Valassi, Combining correlated measurements of several different physical quantities, Nucl. Instrum. Methods Phys. Res., Sect. A 500, 391 (2003).

[33] C. Alexandrou and C. Kallidonis, Low-lying baryon masses using $N_{f}=2$ twisted mass clover-improved fermions directly at the physical pion mass, Phys. Rev. D 96, 034511 (2017). 
R. Aaij, ${ }^{27}$ B. Adeva, ${ }^{41}$ M. Adinolfi, ${ }^{48}$ C. A. Aidala, ${ }^{73}$ Z. Ajaltouni, ${ }^{5}$ S. Akar,${ }^{59}$ P. Albicocco, ${ }^{18}$ J. Albrecht,${ }^{10}$ F. Alessio, ${ }^{42}$ M. Alexander, ${ }^{53}$ A. Alfonso Albero, ${ }^{40} \mathrm{~S}$. Ali, ${ }^{27} \mathrm{G}$. Alkhazov, ${ }^{33}$ P. Alvarez Cartelle, ${ }^{55}$ A. A. Alves Jr, ${ }^{41} \mathrm{~S}$. Amato, ${ }^{2}$ S. Amerio, ${ }^{23}$ Y. Amhis, ${ }^{7}$ L. An,${ }^{3}$ L. Anderlini, ${ }^{17}$ G. Andreassi, ${ }^{43}$ M. Andreotti, ${ }^{16, a}$ J. E. Andrews,${ }^{60}$ R. B. Appleby, ${ }^{56}$ F. Archilli, ${ }^{27}$ P. d'Argent, ${ }^{12}$ J. Arnau Romeu, ${ }^{6}$ A. Artamonov, ${ }^{39}$ M. Artuso, ${ }^{61}$ K. Arzymatov,${ }^{37}$ E. Aslanides, ${ }^{6}$ M. Atzeni, ${ }^{44}$ B. Audurier, ${ }^{22}$ S. Bachmann, ${ }^{12}$ J. J. Back,${ }^{50}$ S. Baker, ${ }^{55}$ V. Balagura, ${ }^{7, b}$ W. Baldini, ${ }^{16}$ A. Baranov, ${ }^{37}$ R. J. Barlow, ${ }^{56}$

S. Barsuk, ${ }^{7}$ W. Barter, ${ }^{56}$ F. Baryshnikov, ${ }^{70}$ V. Batozskaya, ${ }^{31}$ B. Batsukh, ${ }^{61}$ V. Battista, ${ }^{43}$ A. Bay, ${ }^{43}$ J. Beddow ${ }^{53}$ F. Bedeschi, ${ }^{24}$ I. Bediaga, ${ }^{1}$ A. Beiter, ${ }^{61}$ L. J. Bel, ${ }^{27}$ S. Belin, ${ }^{22}$ N. Beliy, ${ }^{63}$ V. Bellee ${ }^{43}$ N. Belloli, ${ }^{20, c}$ K. Belous, ${ }^{39}$ I. Belyaev, ${ }^{34,42}$ E. Ben-Haim, ${ }^{8}$ G. Bencivenni, ${ }^{18}$ S. Benson, ${ }^{27}$ S. Beranek, ${ }^{9}$ A. Berezhnoy, ${ }^{35}$ R. Bernet, ${ }^{44}$ D. Berninghoff, ${ }^{12}$ E. Bertholet, ${ }^{8}$ A. Bertolin, ${ }^{23}$ C. Betancourt, ${ }^{44}$ F. Betti, ${ }^{15,42}$ M. O. Bettler, ${ }^{49}$ M. van Beuzekom, ${ }^{27}$ Ia. Bezshyiko, ${ }^{44}$ S. Bhasin, ${ }^{48}$ J. Bhom, ${ }^{29}$ S. Bifani, ${ }^{47}$ P. Billoir, ${ }^{8}$ A. Birnkraut, ${ }^{10}$ A. Bizzeti,,${ }^{17,}$ M. Bjørn,${ }^{57}$ M. P. Blago, ${ }^{42}$ T. Blake, ${ }^{50}$ F. Blanc, ${ }^{43}$ S. Blusk,${ }^{61}$ D. Bobulska, ${ }^{53}$ V. Bocci, ${ }^{26}$ O. Boente Garcia, ${ }^{41}$ T. Boettcher, ${ }^{58}$ A. Bondar, ${ }^{38, e}$ N. Bondar, ${ }^{33}$ S. Borghi,${ }^{56,42}$ M. Borisyak,${ }^{37}$ M. Borsato, ${ }^{41}$ F. Bossu, ${ }^{7}$ M. Boubdir, ${ }^{9}$ T. J. V. Bowcock, ${ }^{54}$ C. Bozzi,${ }^{16,42}$ S. Braun, ${ }^{12}$ M. Brodski, ${ }^{42}$ J. Brodzicka, ${ }^{29}$ A. Brossa Gonzalo, ${ }^{50}$ D. Brundu, ${ }^{22}$ E. Buchanan, ${ }^{48}$ A. Buonaura, ${ }^{44}$ C. Burr, ${ }^{56}$ A. Bursche, ${ }^{22}$ J. Buytaert, ${ }^{42}$ W. Byczynski, ${ }^{42}$ S. Cadeddu, ${ }^{22}$ H. Cai, ${ }^{64}$ R. Calabrese, ${ }^{16, a}$ R. Calladine, ${ }^{47}$ M. Calvi, ${ }^{20, c}$ M. Calvo Gomez, ${ }^{40, f}$ A. Camboni, ${ }^{40, f}$ P. Campana, ${ }^{18}$ D. H. Campora Perez, ${ }^{42}$ L. Capriotti, ${ }^{56}$ A. Carbone, ${ }^{15, g}$ G. Carboni, ${ }^{25}$ R. Cardinale, ${ }^{19, h}$ A. Cardini, ${ }^{22}$ P. Carniti, ${ }^{20, c}$

L. Carson, ${ }^{52}$ K. Carvalho Akiba, ${ }^{2}$ G. Casse, ${ }^{54}$ L. Cassina, ${ }^{20}$ M. Cattaneo, ${ }^{42}$ G. Cavallero, ${ }^{19, \mathrm{~h}}$ R. Cenci, ${ }^{24, \mathrm{i}}$ D. Chamont, ${ }^{7}$ M. G. Chapman, ${ }^{48}$ M. Charles, ${ }^{8}$ Ph. Charpentier, ${ }^{42}$ G. Chatzikonstantinidis,${ }^{47}$ M. Chefdeville, ${ }^{4}$ V. Chekalina,${ }^{37}$ C. Chen, ${ }^{3}$ S. Chen, ${ }^{22}$ S.-G. Chitic, ${ }^{42}$ V. Chobanova, ${ }^{41}$ M. Chrzaszcz, ${ }^{42}$ A. Chubykin, ${ }^{33}$ P. Ciambrone, ${ }^{18}$ X. Cid Vidal, ${ }^{41}$ G. Ciezarek,${ }^{42}$ P. E. L. Clarke, ${ }^{52}$ M. Clemencic, ${ }^{42}$ H. V. Cliff, ${ }^{49}$ J. Closier, ${ }^{42}$ V. Coco, ${ }^{42}$ J. A. B. Coelho, ${ }^{7}$ J. Cogan, ${ }^{6}$ E. Cogneras, ${ }^{5}$ L. Cojocariu, ${ }^{32}$ P. Collins, ${ }^{42}$ T. Colombo, ${ }^{42}$ A. Comerma-Montells, ${ }^{12}$ A. Contu, ${ }^{22}$ G. Coombs, ${ }^{42}$ S. Coquereau, ${ }^{40}$ G. Corti, ${ }^{42}$ M. Corvo, ${ }^{16, a}$ C. M. Costa Sobral, ${ }^{50}$ B. Couturier, ${ }^{42}$ G. A. Cowan, ${ }^{52}$ D. C. Craik, ${ }^{58}$ A. Crocombe,${ }^{50}$ M. Cruz Torres, ${ }^{1}$ R. Currie ${ }^{52}$ C. D’Ambrosio, ${ }^{42}$ F. Da Cunha Marinho, ${ }^{2}$ C. L. Da Silva, ${ }^{74}$ E. Dall'Occo, ${ }^{27}$ J. Dalseno, ${ }^{48}$ A. Danilina, ${ }^{34}$ A. Davis, ${ }^{3}$ O. De Aguiar Francisco, ${ }^{42}$ K. De Bruyn, ${ }^{42}$ S. De Capua, ${ }^{56}$ M. De Cian, ${ }^{43}$ J. M. De Miranda, ${ }^{1}$ L. De Paula, ${ }^{2}$ M. De Serio, ${ }^{14, j}$ P. De Simone, ${ }^{18}$ C. T. Dean, ${ }^{53}$ D. Decamp, ${ }^{4}$ L. Del Buono, ${ }^{8}$ B. Delaney, ${ }^{49}$ H.-P. Dembinski, ${ }^{11}$ M. Demmer,${ }^{10}$ A. Dendek,${ }^{30}$ D. Derkach, ${ }^{37}$ O. Deschamps, ${ }^{5}$ F. Desse, ${ }^{7}$ F. Dettori, ${ }^{54}$ B. Dey,${ }^{65}$ A. Di Canto, ${ }^{42}$ P. Di Nezza,${ }^{18}$ S. Didenko, ${ }^{70}$ H. Dijkstra, ${ }^{42}$ F. Dordei, ${ }^{42}$ M. Dorigo, ${ }^{42, k}$ A. Dosil Suárez, ${ }^{41}$ L. Douglas, ${ }^{53}$ A. Dovbnya, ${ }^{45}$ K. Dreimanis, ${ }^{54}$ L. Dufour, ${ }^{27}$ G. Dujany, ${ }^{8}$ P. Durante, ${ }^{42}$ J. M. Durham,${ }^{74}$ D. Dutta,${ }^{56}$ R. Dzhelyadin, ${ }^{39}$ M. Dziewiecki, ${ }^{12}$ A. Dziurda, ${ }^{29}$ A. Dzyuba, ${ }^{33}$ S. Easo, ${ }^{51}$ U. Egede ${ }^{55}$ V. Egorychev,${ }^{34}$ S. Eidelman, ${ }^{38, e}$ S. Eisenhardt, ${ }^{52}$ U. Eitschberger, ${ }^{10}$ R. Ekelhof, ${ }^{10}$ L. Eklund, ${ }^{53}$ S. Ely, ${ }^{61}$ A. Ene, ${ }^{32}$ S. Escher, ${ }^{9}$ S. Esen, ${ }^{27}$ T. Evans, ${ }^{59}$ A. Falabella, ${ }^{15}$ N. Farley, ${ }^{47}$ S. Farry, ${ }^{54}$ D. Fazzini, ${ }^{20,42, \mathrm{c}}$ L. Federici,${ }^{25}$ P. Fernandez Declara, ${ }^{42}$ A. Fernandez Prieto, ${ }^{41}$ F. Ferrari, ${ }^{15}$ L. Ferreira Lopes,${ }^{43}$ F. Ferreira Rodrigues, ${ }^{2}$ M. Ferro-Luzzi, ${ }^{42}$ S. Filippov, ${ }^{36}$ R. A. Fini, ${ }^{14}$ M. Fiorini, ${ }^{16, a}$ M. Firlej ${ }^{30}$ C. Fitzpatrick,${ }^{43}$ T. Fiutowski, ${ }^{30}$ F. Fleuret,${ }^{7, b}$ M. Fontana, ${ }^{22,42}$ F. Fontanelli, ${ }^{19, \mathrm{~h}}$ R. Forty ${ }^{42}$ V. Franco Lima, ${ }^{54}$ M. Frank,${ }^{42}$ C. Frei, ${ }^{42}$ J. Fu, ${ }^{21,1}$ W. Funk, ${ }^{42}$ C. Färber,${ }^{42}$

M. Féo Pereira Rivello Carvalho, ${ }^{27}$ E. Gabriel, ${ }^{52}$ A. Gallas Torreira, ${ }^{41}$ D. Galli, ${ }^{15, g}$ S. Gallorini, ${ }^{23}$ S. Gambetta, ${ }^{52}$ Y. Gan, ${ }^{3}$ M. Gandelman, ${ }^{2}$ P. Gandini, ${ }^{21}$ Y. Gao, ${ }^{3}$ L. M. Garcia Martin, ${ }^{72}$ B. Garcia Plana, ${ }^{41}$ J. García Pardiñas, ${ }^{44}$ J. Garra Tico, ${ }^{49}$ L. Garrido, ${ }^{40}$ D. Gascon, ${ }^{40}$ C. Gaspar, ${ }^{42}$ L. Gavardi,${ }^{10}$ G. Gazzoni, ${ }^{5}$ D. Gerick, ${ }^{12}$ E. Gersabeck, ${ }^{56}$ M. Gersabeck, ${ }^{56}$ T. Gershon, ${ }^{50}$ D. Gerstel, ${ }^{6}$ Ph. Ghez, ${ }^{4}$ S. Gianì, ${ }^{43}$ V. Gibson, ${ }^{49}$ O. G. Girard, ${ }^{43}$ L. Giubega, ${ }^{32}$ K. Gizdov, ${ }^{52}$ V. V. Gligorov, ${ }^{8}$ D. Golubkov, ${ }^{34}$ A. Golutvin, ${ }^{55,70}$ A. Gomes, ${ }^{1, \mathrm{~m}}$ I. V. Gorelov,${ }^{35}$ C. Gotti, ${ }^{20, \mathrm{c}}$ E. Govorkova, ${ }^{27}$ J. P. Grabowski, ${ }^{12}$

R. Graciani Diaz, ${ }^{40}$ L. A. Granado Cardoso, ${ }^{42}$ E. Graugés, ${ }^{40}$ E. Graverini, ${ }^{44}$ G. Graziani, ${ }^{17}$ A. Grecu, ${ }^{32}$ R. Greim, ${ }^{27}$ P. Griffith, ${ }^{22}$ L. Grillo, ${ }^{56}$ L. Gruber, ${ }^{42}$ B. R. Gruberg Cazon, ${ }^{57}$ O. Grünberg, ${ }^{67}$ C. Gu, ${ }^{3}$ E. Gushchin, ${ }^{36}$ Yu. Guz, ${ }^{39,42}$ T. Gys,${ }^{42}$ C. Göbel, ${ }^{62}$ T. Hadavizadeh, ${ }^{57}$ C. Hadjivasiliou, ${ }^{5}$ G. Haefeli, ${ }^{43}$ C. Haen, ${ }^{42}$ S. C. Haines, ${ }^{49}$ B. Hamilton ${ }^{60}$ X. Han, ${ }^{12}$ T. H. Hancock ${ }^{57}$ S. Hansmann-Menzemer, ${ }^{12}$ N. Harnew, ${ }^{57}$ S. T. Harnew, ${ }^{48}$ T. Harrison, ${ }^{54}$ C. Hasse, ${ }^{42}$ M. Hatch,${ }^{42}$ J. He ${ }^{63}$ M. Hecker ${ }^{55}$ K. Heinicke, ${ }^{10}$ A. Heister, ${ }^{10}$ K. Hennessy, ${ }^{54}$ L. Henry, ${ }^{72}$ E. van Herwijnen, ${ }^{42}$ M. Heß,${ }^{67}$ A. Hicheur, ${ }^{2}$ R. Hidalgo Charman ${ }^{56}$ D. Hill, ${ }^{57}$ M. Hilton, ${ }^{56}$ P. H. Hopchev,${ }^{43}$ W. Hu,${ }^{65}$ W. Huang, ${ }^{63}$ Z. C. Huard ${ }^{59}$ W. Hulsbergen, ${ }^{27}$ T. Humair ${ }^{55}$ M. Hushchyn, ${ }^{37}$ D. Hutchcroft, ${ }^{54}$ D. Hynds, ${ }^{27}$ P. Ibis, ${ }^{10}$ M. Idzik, ${ }^{30}$ P. Ilten, ${ }^{47}$ K. Ivshin, ${ }^{33}$ R. Jacobsson, ${ }^{42}$ J. Jalocha, ${ }^{57}$ E. Jans, ${ }^{27}$ A. Jawahery, ${ }^{60}$ F. Jiang, ${ }^{3}$ M. John, ${ }^{57}$ D. Johnson, ${ }^{42}$ C. R. Jones, ${ }^{49}$ C. Joram, ${ }^{42}$ B. Jost, ${ }^{42}$ N. Jurik, ${ }^{57}$ S. Kandybei ${ }^{45}$ M. Karacson, ${ }^{42}$ J. M. Kariuki, ${ }^{48}$ S. Karodia, ${ }^{53}$ N. Kazeev,${ }^{37}$ M. Kecke, ${ }^{12}$ F. Keizer, ${ }^{49}$ M. Kelsey, ${ }^{61}$ M. Kenzie, ${ }^{49}$ T. Ketel, ${ }^{28}$ E. Khairullin, ${ }^{37}$ B. Khanji, ${ }^{12}$ C. Khurewathanakul, ${ }^{43}$ K. E. Kim, ${ }^{61}$ T. Kirn, ${ }^{9}$ S. Klaver, ${ }^{18}$ K. Klimaszewski, ${ }^{31}$ T. Klimkovich, ${ }^{11}$ S. Koliiev, ${ }^{46}$ M. Kolpin, ${ }^{12}$ R. Kopecna, ${ }^{12}$ P. Koppenburg, ${ }^{27}$ I. Kostiuk, ${ }^{27}$ S. Kotriakhova, ${ }^{33}$ M. Kozeiha, ${ }^{5}$ L. Kravchuk, ${ }^{36}$ M. Kreps,${ }^{50}$ F. Kress, ${ }^{55}$ P. Krokovny, ${ }^{38, e}$ W. Krupa, ${ }^{30}$ W. Krzemien, ${ }^{31}$ 
W. Kucewicz, ${ }^{29, \mathrm{n}}$ M. Kucharczyk, ${ }^{29}$ V. Kudryavtsev, ${ }^{38, \mathrm{e}}$ A. K. Kuonen, ${ }^{43}$ T. Kvaratskheliya, ${ }^{34,42}$ D. Lacarrere, ${ }^{42}$ G. Lafferty, ${ }^{56}$ A. Lai, ${ }^{22}$ D. Lancierini, ${ }^{44}$ G. Lanfranchi, ${ }^{18}$ C. Langenbruch,${ }^{9}$ T. Latham,${ }^{50}$ C. Lazzeroni, ${ }^{47}$ R. Le Gac, ${ }^{6}$ A. Leflat, ${ }^{35}$ J. Lefrançois, ${ }^{7}$ R. Lefèvre, ${ }^{5}$ F. Lemaitre, ${ }^{42}$ O. Leroy, ${ }^{6}$ T. Lesiak, ${ }^{29}$ B. Leverington, ${ }^{12}$ P.-R. Li ${ }^{63}$ T. Li, ${ }^{3}$ Z. Li, ${ }^{61}$ X. Liang, ${ }^{61}$ T. Likhomanenko, ${ }^{69}$ R. Lindner, ${ }^{42}$ F. Lionetto, ${ }^{44}$ V. Lisovskyi, ${ }^{7}$ X. Liu, ${ }^{3}$ D. Loh, ${ }^{50}$ A. Loi,${ }^{22}$ I. Longstaff, ${ }^{53}$ J. H. Lopes, ${ }^{2}$ G. H. Lovell, ${ }^{49}$ D. Lucchesi ${ }^{23,0}$ M. Lucio Martinez, ${ }^{41}$ A. Lupato, ${ }^{23}$ E. Luppi, ${ }^{16, a}$ O. Lupton, ${ }^{42}$ A. Lusiani, ${ }^{24}$ X. Lyu ${ }^{63}$ F. Machefert, ${ }^{7}$ F. Maciuc ${ }^{32}$ V. Macko, ${ }^{43}$ P. Mackowiak, ${ }^{10}$ S. Maddrell-Mander, ${ }^{48}$ O. Maev, ${ }^{33,42}$ K. Maguire ${ }^{56}$ D. Maisuzenko, ${ }^{33}$ M. W. Majewski,${ }^{30}$ S. Malde,${ }^{57}$ B. Malecki, ${ }^{29}$ A. Malinin, ${ }^{69}$ T. Maltsev, ${ }^{38, e}$ G. Manca, ${ }^{22, p}$ G. Mancinelli, ${ }^{6}$ D. Marangotto, ${ }^{21,1}$ J. Maratas, ${ }^{5, q}$ J. F. Marchand, ${ }^{4}$ U. Marconi, ${ }^{15}$ C. Marin Benito, ${ }^{7}$ M. Marinangeli, ${ }^{43}$ P. Marino, ${ }^{43}$ J. Marks, ${ }^{12}$ P. J. Marshall, ${ }^{54}$ G. Martellotti, ${ }^{26}$ M. Martin, ${ }^{6}$ M. Martinelli, ${ }^{42}$ D. Martinez Santos, ${ }^{41}$ F. Martinez Vidal, ${ }^{72}$ A. Massafferri, ${ }^{1}$ M. Materok, ${ }^{9}$ R. Matev ${ }^{42}$ A. Mathad,${ }^{50}$ Z. Mathe,${ }^{42}$ C. Matteuzzi, ${ }^{20}$ A. Mauri, ${ }^{44}$ E. Maurice, ${ }^{7, b}$ B. Maurin, ${ }^{43}$ A. Mazurov, ${ }^{47}$ M. McCann,${ }^{55,42}$ A. McNab,${ }^{56}$ R. McNulty, ${ }^{13}$ J. V. Mead, ${ }^{54}$ B. Meadows, ${ }^{59}$ C. Meaux, ${ }^{6}$ F. Meier, ${ }^{10}$ N. Meinert ${ }^{67}$ D. Melnychuk ${ }^{31}$ M. Merk,${ }^{27}$ A. Merli, ${ }^{21,1}$ E. Michielin, ${ }^{23}$ D. A. Milanes ${ }^{66}$ E. Millard, ${ }^{50}$ M.-N. Minard, L. Minzoni, ${ }^{16, a}$ D. S. Mitzel, ${ }^{12}$ A. Mogini, ${ }^{8}$ J. Molina Rodriguez, ${ }^{1, r}$ T. Mombächer, ${ }^{10}$ I. A. Monroy, ${ }^{66}$ S. Monteil, ${ }^{5}$ M. Morandin, ${ }^{23}$ G. Morello, ${ }^{18}$ M. J. Morello, ${ }^{24, s}$ O. Morgunova, ${ }^{69}$ J. Moron, ${ }^{30}$ A. B. Morris,${ }^{6}$ R. Mountain, ${ }^{61}$ F. Muheim, ${ }^{52}$ M. Mulder ${ }^{27}$ C. H. Murphy, ${ }^{57}$ D. Murray,${ }^{56}$ A. Mödden, ${ }^{10}$ D. Müller, ${ }^{42}$ J. Müller, ${ }^{10}$ K. Müller,${ }^{44}$ V. Müller ${ }^{10}$ P. Naik, ${ }^{48}$ T. Nakada, ${ }^{43}$ R. Nandakumar, ${ }^{51}$ A. Nandi, ${ }^{57}$ T. Nanut, ${ }^{43}$ I. Nasteva, ${ }^{2}$ M. Needham,${ }^{52}$ N. Neri, ${ }^{21}$ S. Neubert, ${ }^{12}$ N. Neufeld,${ }^{42}$ M. Neuner, ${ }^{12}$ T. D. Nguyen,${ }^{43}$ C. Nguyen-Mau, ${ }^{43, t}$ S. Nieswand, ${ }^{9}$ R. Niet, ${ }^{10}$ N. Nikitin, ${ }^{35}$ A. Nogay, ${ }^{69}$ N. S. Nolte, ${ }^{42}$ D. P. O'Hanlon, ${ }^{15}$ A. Oblakowska-Mucha, ${ }^{30}$ V. Obraztsov, ${ }^{39}$ S. Ogilvy, ${ }^{18}$ R. Oldeman, ${ }^{22, p}$ C. J. G. Onderwater,${ }^{68}$ A. Ossowska, ${ }^{29}$ J. M. Otalora Goicochea, ${ }^{2}$ P. Owen, ${ }^{44}$ A. Oyanguren, ${ }^{72}$ P. R. Pais, ${ }^{43}$ T. Pajero, ${ }^{24, s}$ A. Palano, ${ }^{14}$ M. Palutan, ${ }^{18,42}$ G. Panshin,${ }^{71}$ A. Papanestis,${ }^{51}$ M. Pappagallo, ${ }^{52}$ L. L. Pappalardo, ${ }^{16, a}$ W. Parker,${ }^{60}$ C. Parkes, ${ }^{56}$ G. Passaleva, ${ }^{17,42}$ A. Pastore, ${ }^{14}$ M. Patel,${ }^{55}$ C. Patrignani, ${ }^{15,9}$ A. Pearce, ${ }^{42}$ A. Pellegrino, ${ }^{27}$ G. Penso, ${ }^{26}$ M. Pepe Altarelli, ${ }^{42}$ S. Perazzini ${ }^{42}$ D. Pereima ${ }^{34}$ P. Perret,${ }^{5}$ L. Pescatore, ${ }^{43}$ K. Petridis,${ }^{48}$ A. Petrolini, ${ }^{19, h}$ A. Petrov,${ }^{69}$ S. Petrucci, ${ }^{52}$ M. Petruzzo,${ }^{21,1}$ B. Pietrzyk, ${ }^{4}$ G. Pietrzyk ${ }^{43}$ M. Pikies, ${ }^{29}$ M. Pili, ${ }^{57}$ D. Pinci, ${ }^{26}$ J. Pinzino, ${ }^{42}$ F. Pisani, ${ }^{42}$ A. Piucci, ${ }^{12}$ V. Placinta, ${ }^{32}$ S. Playfer, ${ }^{52}$ J. Plews, ${ }^{47}$ M. Plo Casasus, ${ }^{41}$ F. Polci, ${ }^{8}$ M. Poli Lener, ${ }^{18}$ A. Poluektov, ${ }^{50}$ N. Polukhina, ${ }^{70, u}$ I. Polyakov, ${ }^{61}$ E. Polycarpo, ${ }^{2}$ G. J. Pomery ${ }^{48}$ S. Ponce, ${ }^{42}$ A. Popov,${ }^{39}$ D. Popov,${ }^{47,11}$ S. Poslavskii, ${ }^{39}$ C. Potterat, ${ }^{2}$ E. Price, ${ }^{48}$ J. Prisciandaro, ${ }^{41}$ C. Prouve, ${ }^{48}$ V. Pugatch,${ }^{46}$ A. Puig Navarro, ${ }^{44}$ H. Pullen, ${ }^{57}$ G. Punzi, ${ }^{24, i}$ W. Qian, ${ }^{63}$ J. Qin, ${ }^{63}$ R. Quagliani, ${ }^{8}$ B. Quintana, ${ }^{5}$ B. Rachwal, ${ }^{30}$ J. H. Rademacker, ${ }^{48}$ M. Rama, ${ }^{24}$ M. Ramos Pernas, ${ }^{41}$ M. S. Rangel, ${ }^{2}$ F. Ratnikov, ${ }^{37, v}$ G. Raven, ${ }^{28}$ M. Ravonel Salzgeber, ${ }^{42}$ M. Reboud, ${ }^{4}$ F. Redi, ${ }^{43}$ S. Reichert, ${ }^{10}$ A. C. dos Reis, ${ }^{1}$ F. Reiss,${ }^{8}$ C. Remon Alepuz, ${ }^{72}$ Z. Ren, ${ }^{3}$ V. Renaudin, ${ }^{7}$ S. Ricciardi, ${ }^{51}$ S. Richards,${ }^{48}$ K. Rinnert, ${ }^{54}$ P. Robbe, ${ }^{7}$ A. Robert, ${ }^{8}$ A. B. Rodrigues, ${ }^{43}$ E. Rodrigues,${ }^{59}$ J. A. Rodriguez Lopez, ${ }^{66}$ M. Roehrken, ${ }^{42}$ A. Rogozhnikov, ${ }^{37}$ S. Roiser, ${ }^{42}$ A. Rollings, ${ }^{57}$ V. Romanovskiy, ${ }^{39}$

A. Romero Vidal, ${ }^{41}$ M. Rotondo, ${ }^{18}$ M. S. Rudolph ${ }^{61}$ T. Ruf, ${ }^{42}$ J. Ruiz Vidal, ${ }^{72}$ J. J. Saborido Silva, ${ }^{41}$ N. Sagidova, ${ }^{33}$

B. Saitta, ${ }^{22, p}$ V. Salustino Guimaraes, ${ }^{62}$ C. Sanchez Gras, ${ }^{27}$ C. Sanchez Mayordomo, ${ }^{72}$ B. Sanmartin Sedes, ${ }^{41}$

R. Santacesaria, ${ }^{26}$ C. Santamarina Rios, ${ }^{41}$ M. Santimaria, ${ }^{18}$ E. Santovetti, ${ }^{25, w}$ G. Sarpis, ${ }^{56}$ A. Sarti, ${ }^{18, x}$ C. Satriano, ${ }^{26, y}$ A. Satta, ${ }^{25}$ M. Saur, ${ }^{63}$ D. Savrina, ${ }^{34,35}$ S. Schael, ${ }^{9}$ M. Schellenberg, ${ }^{10}$ M. Schiller, ${ }^{53}$ H. Schindler ${ }^{42}$ M. Schmelling, ${ }^{11}$ T. Schmelzer, ${ }^{10}$ B. Schmidt, ${ }^{42}$ O. Schneider, ${ }^{43}$ A. Schopper, ${ }^{42}$ H. F. Schreiner, ${ }^{59}$ M. Schubiger, ${ }^{43}$ M. H. Schune, ${ }^{7}$ R. Schwemmer, ${ }^{42}$ B. Sciascia, ${ }^{18}$ A. Sciubba, ${ }^{26, x}$ A. Semennikov, ${ }^{34}$ E. S. Sepulveda, ${ }^{8}$ A. Sergi, ${ }^{47,42}$ N. Serra, ${ }^{44}$ J. Serrano, ${ }^{6}$ L. Sestini, ${ }^{23}$ A. Seuthe, ${ }^{10}$ P. Seyfert, ${ }^{42}$ M. Shapkin, ${ }^{39}$ Y. Shcheglov, ${ }^{33, \dagger}$ T. Shears, ${ }^{54}$ L. Shekhtman, ${ }^{38, e}$ V. Shevchenko, ${ }^{69}$ E. Shmanin, ${ }^{70}$ B. G. Siddi, ${ }^{16}$ R. Silva Coutinho, ${ }^{44}$ L. Silva de Oliveira, ${ }^{2}$ G. Simi,${ }^{23,0}$ S. Simone, ${ }^{14, j}$ N. Skidmore, ${ }^{12}$ T. Skwarnicki, ${ }^{61}$ J. G. Smeaton, ${ }^{49}$ E. Smith, ${ }^{9}$ I. T. Smith, ${ }^{52}$ M. Smith,${ }^{55}$ M. Soares, ${ }^{15}$ 1. Soares Lavra, ${ }^{1}$ M. D. Sokoloff, ${ }^{59}$ F. J. P. Soler, ${ }^{53}$ B. Souza De Paula, ${ }^{2}$ B. Spaan, ${ }^{10}$ P. Spradlin,${ }^{53}$ F. Stagni, ${ }^{42}$ M. Stahl,${ }^{12}$ S. Stahl,${ }^{42}$ P. Stefko, ${ }^{43}$ S. Stefkova ${ }^{55}$ O. Steinkamp, ${ }^{44}$ S. Stemmle, ${ }^{12}$ O. Stenyakin, ${ }^{39}$ M. Stepanova ${ }^{33}$ H. Stevens, ${ }^{10}$ A. Stocchi, ${ }^{7}$ S. Stone,${ }^{61}$ B. Storaci, ${ }^{44}$ S. Stracka, ${ }^{24, i}$ M. E. Stramaglia, ${ }^{43}$ M. Straticiuc, ${ }^{32}$ U. Straumann, ${ }^{44}$ S. Strokov, ${ }^{71}$ J. Sun, ${ }^{3}$ L. Sun, ${ }^{64}$ K. Swientek, ${ }^{30}$ V. Syropoulos,${ }^{28}$ T. Szumlak,${ }^{30}$ M. Szymanski ${ }^{63}$ S. T'Jampens, ${ }^{4}$ Z. Tang, ${ }^{3}$ A. Tayduganov, ${ }^{6}$ T. Tekampe,${ }^{10}$ G. Tellarini, ${ }^{16}$ F. Teubert, ${ }^{42}$ E. Thomas, ${ }^{42}$ J. van Tilburg, ${ }^{27}$ M. J. Tilley ${ }^{55}$ V. Tisserand, ${ }^{5}$ M. Tobin,${ }^{30}$ S. Tolk, ${ }^{42}$ L. Tomassetti, ${ }^{16, a}$

D. Tonelli, ${ }^{24}$ D. Y. Tou,${ }^{8}$ R. Tourinho Jadallah Aoude, ${ }^{1}$ E. Tournefier, ${ }^{4}$ M. Traill,${ }^{53}$ M. T. Tran, ${ }^{43}$ A. Trisovic, ${ }^{49}$

A. Tsaregorodtsev, ${ }^{6}$ G. Tuci, ${ }^{24}$ A. Tully, ${ }^{49}$ N. Tuning, ${ }^{27,42}$ A. Ukleja, ${ }^{31}$ A. Usachov, ${ }^{7}$ A. Ustyuzhanin, ${ }^{37}$ U. Uwer, ${ }^{12}$ A. Vagner,${ }^{71}$ V. Vagnoni, ${ }^{15}$ A. Valassi ${ }^{42}$ S. Valat ${ }^{42}$ G. Valenti, ${ }^{15}$ R. Vazquez Gomez, ${ }^{42}$ P. Vazquez Regueiro, ${ }^{41}$ S. Vecchi, ${ }^{16}$ M. van Veghel, ${ }^{27}$ J. J. Velthuis, ${ }^{48}$ M. Veltri, ${ }^{17, z}$ G. Veneziano, ${ }^{57}$ A. Venkateswaran, ${ }^{61}$ T. A. Verlage, ${ }^{9}$ M. Vernet, ${ }^{5}$ M. Veronesi, ${ }^{27}$ N. V. Veronika, ${ }^{13}$ M. Vesterinen, ${ }^{57}$ J. V. Viana Barbosa, ${ }^{42}$ D. Vieira, ${ }^{63}$ M. Vieites Diaz, ${ }^{41}$ H. Viemann, ${ }^{67}$ 
X. Vilasis-Cardona, ${ }^{40, f}$ A. Vitkovskiy, ${ }^{27}$ M. Vitti ${ }^{49}$ V. Volkov, ${ }^{35}$ A. Vollhardt,${ }^{44}$ B. Voneki, ${ }^{42}$ A. Vorobyev, ${ }^{33}$ V. Vorobyev, ${ }^{38, e}$ J. A. de Vries, ${ }^{27}$ C. Vázquez Sierra, ${ }^{27}$ R. Waldi, ${ }^{67}$ J. Walsh, ${ }^{24}$ J. Wang, ${ }^{61}$ M. Wang, ${ }^{3}$ Y. Wang, ${ }^{65}$ Z. Wang, ${ }^{44}$ D. R. Ward, ${ }^{49}$ H. M. Wark, ${ }^{54}$ N. K. Watson, ${ }^{47}$ D. Websdale ${ }^{55}$ A. Weiden, ${ }^{44}$ C. Weisser, ${ }^{58}$ M. Whitehead, ${ }^{9}$ J. Wicht,${ }^{50}$ G. Wilkinson, ${ }^{57}$ M. Wilkinson, ${ }^{61}$ I. Williams, ${ }^{49}$ M. R. J. Williams, ${ }^{56}$ M. Williams, ${ }^{58}$ T. Williams, ${ }^{47}$ F. F. Wilson, ${ }^{51,42}$ J. Wimberley,${ }^{60}$ M. Winn, ${ }^{7}$ J. Wishahi, ${ }^{10}$ W. Wislicki,${ }^{31}$ M. Witek, ${ }^{29}$ G. Wormser, ${ }^{7}$ S. A. Wotton, ${ }^{49}$ K. Wyllie,${ }^{42}$ D. Xiao, ${ }^{65}$ Y. Xie, ${ }^{65}$ A. Xu,${ }^{3}$

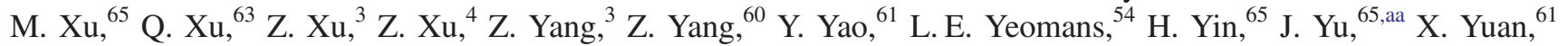
O. Yushchenko, ${ }^{39}$ K. A. Zarebski, ${ }^{47}$ M. Zavertyaev, ${ }^{11, u}$ D. Zhang, ${ }^{65}$ L. Zhang, ${ }^{3}$ W. C. Zhang, ${ }^{3, b b}$ Y. Zhang, ${ }^{7}$ A. Zhelezov, ${ }^{12}$ Y. Zheng, ${ }^{63}$ X. Zhu, ${ }^{3}$ V. Zhukov, ${ }^{9,35}$ J. B. Zonneveld, ${ }^{52}$ and S. Zucchelli ${ }^{15}$

\title{
(LHCb Collaboration)
}

\author{
${ }^{1}$ Centro Brasileiro de Pesquisas Físicas (CBPF), Rio de Janeiro, Brazil \\ ${ }^{2}$ Universidade Federal do Rio de Janeiro (UFRJ), Rio de Janeiro, Brazil \\ ${ }^{3}$ Center for High Energy Physics, Tsinghua University, Beijing, China \\ ${ }^{4}$ Univ. Grenoble Alpes, Univ. Savoie Mont Blanc, CNRS, IN2P3-LAPP, Annecy, France \\ ${ }^{5}$ Clermont Université, Université Blaise Pascal, CNRS/IN2P3, LPC, Clermont-Ferrand, France \\ ${ }^{6}$ Aix Marseille Univ, CNRS/IN2P3, CPPM, Marseille, France \\ ${ }^{7}$ LAL, Univ. Paris-Sud, CNRS/IN2P3, Université Paris-Saclay, Orsay, France \\ ${ }^{8}$ LPNHE, Sorbonne Université, Paris Diderot Sorbonne Paris Cité, CNRS/IN2P3, Paris, France \\ ${ }^{9}$ I. Physikalisches Institut, RWTH Aachen University, Aachen, Germany \\ ${ }^{10}$ Fakultät Physik, Technische Universität Dortmund, Dortmund, Germany \\ ${ }^{11}$ Max-Planck-Institut für Kernphysik (MPIK), Heidelberg, Germany \\ ${ }^{12}$ Physikalisches Institut, Ruprecht-Karls-Universität Heidelberg, Heidelberg, Germany \\ ${ }^{13}$ School of Physics, University College Dublin, Dublin, Ireland \\ ${ }^{14}$ INFN Sezione di Bari, Bari, Italy \\ ${ }^{15}$ INFN Sezione di Bologna, Bologna, Italy \\ ${ }^{16}$ INFN Sezione di Ferrara, Ferrara, Italy \\ ${ }^{17}$ INFN Sezione di Firenze, Firenze, Italy \\ ${ }^{18}$ INFN Laboratori Nazionali di Frascati, Frascati, Italy \\ ${ }^{19}$ INFN Sezione di Genova, Genova, Italy \\ ${ }^{20}$ INFN Sezione di Milano-Bicocca, Milano, Italy \\ ${ }^{21}$ INFN Sezione di Milano, Milano, Italy \\ ${ }^{22}$ INFN Sezione di Cagliari, Monserrato, Italy \\ ${ }^{23}$ INFN Sezione di Padova, Padova, Italy \\ ${ }^{24}$ INFN Sezione di Pisa, Pisa, Italy \\ ${ }^{25}$ INFN Sezione di Roma Tor Vergata, Roma, Italy \\ ${ }^{26}$ INFN Sezione di Roma La Sapienza, Roma, Italy \\ ${ }^{27}$ Nikhef National Institute for Subatomic Physics, Amsterdam, Netherlands \\ ${ }^{28}$ Nikhef National Institute for Subatomic Physics and VU University Amsterdam, Amsterdam, Netherlands \\ ${ }^{29}$ Henryk Niewodniczanski Institute of Nuclear Physics Polish Academy of Sciences, Kraków, Poland \\ ${ }^{30}$ AGH - University of Science and Technology, Faculty of Physics and Applied Computer Science, Kraków, Poland \\ ${ }^{31}$ National Center for Nuclear Research (NCBJ), Warsaw, Poland \\ ${ }^{32}$ Horia Hulubei National Institute of Physics and Nuclear Engineering, Bucharest-Magurele, Romania \\ ${ }^{33}$ Petersburg Nuclear Physics Institute (PNPI), Gatchina, Russia \\ ${ }^{34}$ Institute of Theoretical and Experimental Physics (ITEP), Moscow, Russia \\ ${ }^{35}$ Institute of Nuclear Physics, Moscow State University (SINP MSU), Moscow, Russia \\ ${ }^{36}$ Institute for Nuclear Research of the Russian Academy of Sciences (INR RAS), Moscow, Russia \\ ${ }^{37}$ Yandex School of Data Analysis, Moscow, Russia \\ ${ }^{38}$ Budker Institute of Nuclear Physics (SB RAS), Novosibirsk, Russia \\ ${ }^{39}$ Institute for High Energy Physics (IHEP), Protvino, Russia \\ ${ }^{40}$ ICCUB, Universitat de Barcelona, Barcelona, Spain \\ ${ }^{41}$ Instituto Galego de Física de Altas Enerxías (IGFAE), Universidade de Santiago de Compostela, Santiago de Compostela, Spain \\ ${ }^{42}$ European Organization for Nuclear Research (CERN), Geneva, Switzerland \\ ${ }^{43}$ Institute of Physics, Ecole Polytechnique Fédérale de Lausanne (EPFL), Lausanne, Switzerland \\ ${ }^{44}$ Physik-Institut, Universität Zürich, Zürich, Switzerland \\ ${ }^{45}$ NSC Kharkiv Institute of Physics and Technology (NSC KIPT), Kharkiv, Ukraine \\ ${ }^{46}$ Institute for Nuclear Research of the National Academy of Sciences (KINR), Kyiv, Ukraine
}




\author{
${ }^{47}$ University of Birmingham, Birmingham, United Kingdom \\ ${ }^{48}$ H.H. Wills Physics Laboratory, University of Bristol, Bristol, United Kingdom \\ ${ }^{49}$ Cavendish Laboratory, University of Cambridge, Cambridge, United Kingdom \\ ${ }^{50}$ Department of Physics, University of Warwick, Coventry, United Kingdom \\ ${ }^{51}$ STFC Rutherford Appleton Laboratory, Didcot, United Kingdom \\ ${ }^{52}$ School of Physics and Astronomy, University of Edinburgh, Edinburgh, United Kingdom \\ ${ }^{53}$ School of Physics and Astronomy, University of Glasgow, Glasgow, United Kingdom \\ ${ }^{54}$ Oliver Lodge Laboratory, University of Liverpool, Liverpool, United Kingdom \\ ${ }^{55}$ Imperial College London, London, United Kingdom \\ ${ }^{56}$ School of Physics and Astronomy, University of Manchester, Manchester, United Kingdom \\ ${ }^{57}$ Department of Physics, University of Oxford, Oxford, United Kingdom \\ ${ }^{58}$ Massachusetts Institute of Technology, Cambridge, Massachusetts, USA \\ ${ }^{59}$ University of Cincinnati, Cincinnati, Ohio, USA \\ ${ }^{60}$ University of Maryland, College Park, Maryland, USA \\ ${ }^{61}$ Syracuse University, Syracuse, New York, USA \\ ${ }^{62}$ Pontifícia Universidade Católica do Rio de Janeiro (PUC-Rio), Rio de Janeiro, Brazil \\ [associated with Universidade Federal do Rio de Janeiro (UFRJ), Rio de Janeiro, Brazil] \\ ${ }^{63}$ University of Chinese Academy of Sciences, Beijing, China \\ (associated with Center for High Energy Physics, Tsinghua University, Beijing, China) \\ ${ }^{64}$ School of Physics and Technology, Wuhan University, Wuhan, China \\ (associated with Center for High Energy Physics, Tsinghua University, Beijing, China) \\ ${ }^{65}$ Institute of Particle Physics, Central China Normal University, Wuhan, Hubei, China \\ (associated with Center for High Energy Physics, Tsinghua University, Beijing, China) \\ ${ }^{66}$ Departamento de Fisica, Universidad Nacional de Colombia, Bogota, Colombia \\ (associated with LPNHE, Sorbonne Université, Paris Diderot Sorbonne Paris Cité, CNRS/IN2P3, Paris, France) \\ ${ }^{67}$ Institut für Physik, Universität Rostock, Rostock, Germany \\ (associated with Physikalisches Institut, Ruprecht-Karls-Universität Heidelberg, Heidelberg, Germany) \\ ${ }^{68}$ Van Swinderen Institute, University of Groningen, Groningen, Netherlands \\ (associated with Nikhef National Institute for Subatomic Physics, Amsterdam, Netherlands) \\ ${ }^{69}$ National Research Centre Kurchatov Institute, Moscow, Russia \\ [associated with Institute of Theoretical and Experimental Physics (ITEP), Moscow, Russia] \\ ${ }^{70}$ National University of Science and Technology "MISIS", Moscow, Russia \\ [associated with Institute of Theoretical and Experimental Physics (ITEP), Moscow, Russia] \\ ${ }^{71}$ National Research Tomsk Polytechnic University, Tomsk, Russia \\ [associated with Institute of Theoretical and Experimental Physics (ITEP), Moscow, Russia] \\ ${ }^{72}$ Instituto de Fisica Corpuscular, Centro Mixto Universidad de Valencia - CSIC, Valencia, Spain \\ (associated with ICCUB, Universitat de Barcelona, Barcelona, Spain) \\ ${ }^{73}$ University of Michigan, Ann Arbor, Michigan, USA \\ (associated with Syracuse University, Syracuse, New York, USA) \\ ${ }^{74}$ Los Alamos National Laboratory (LANL), Los Alamos, New Mexico, USA \\ (associated with Syracuse University, Syracuse, New York, USA)
}

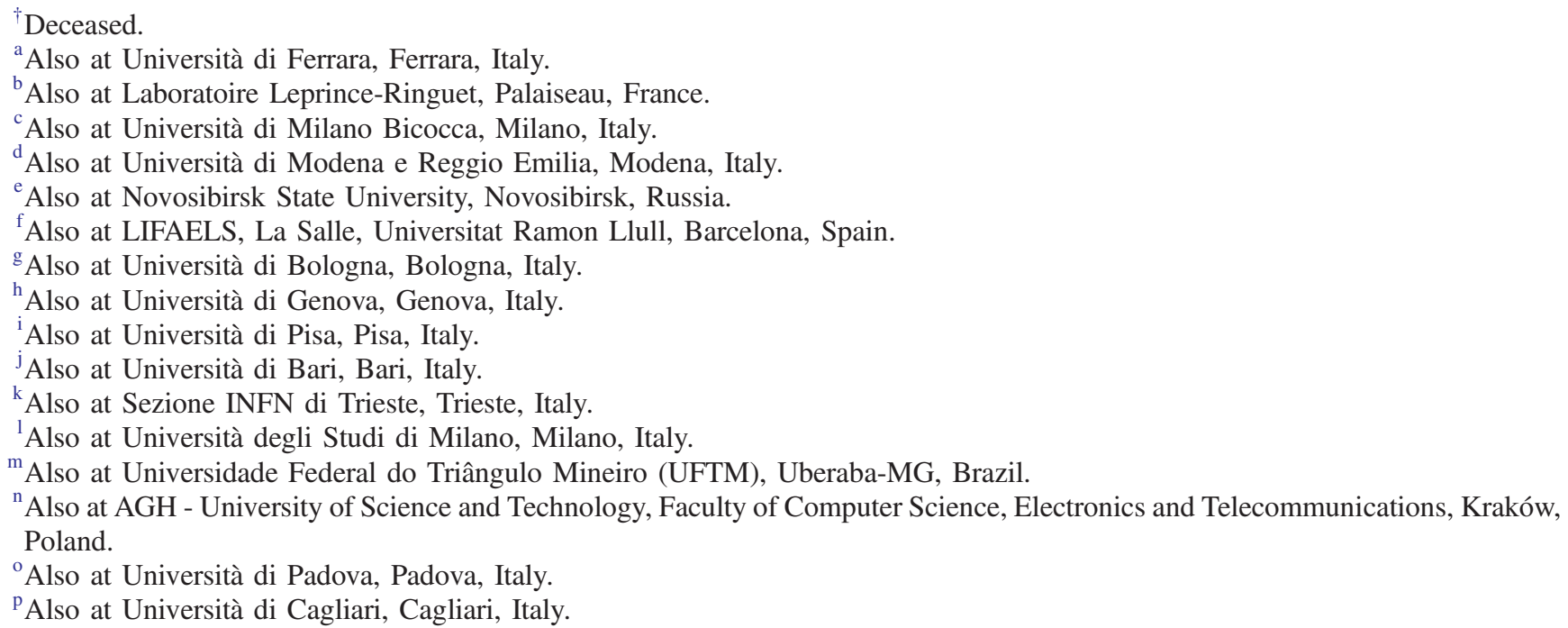


${ }^{\mathrm{q}}$ Also at MSU - Iligan Institute of Technology (MSU-IIT), Iligan, Philippines.

${ }^{\mathrm{r}}$ Also at Escuela Agrícola Panamericana, San Antonio de Oriente, Honduras.

${ }^{\mathrm{s}}$ Also at Scuola Normale Superiore, Pisa, Italy.

${ }^{\mathrm{t}}$ Also at Hanoi University of Science, Hanoi, Vietnam.

"Also at P.N. Lebedev Physical Institute, Russian Academy of Science (LPI RAS), Moscow, Russia.

${ }^{v}$ Also at National Research University Higher School of Economics, Moscow, Russia.

${ }^{\mathrm{w}}$ Also at Università di Roma Tor Vergata, Roma, Italy.

${ }^{x}$ Also at Università di Roma La Sapienza, Roma, Italy.

${ }^{y}$ Also at Università della Basilicata, Potenza, Italy.

${ }^{\mathrm{z}}$ Also at Università di Urbino, Urbino, Italy.

${ }^{\text {aa }}$ Also at Physics and Micro Electronic College, Hunan University, Changsha City, China.

${ }^{\mathrm{bb}}$ Also at School of Physics and Information Technology, Shaanxi Normal University (SNNU), Xi'an, China. 